11,12

\title{
Полиморфизм $\mathrm{ScBO}_{3}$ при твердофазном синтезе из аморфных прекурсоров
}

\author{
() И.М. Шмытько \\ Институт физики твердого тела РАН, \\ Черноголовка, Московская обл. \\ E-mail: shim@issp.ac.ru \\ Поступила в Редакцию 20 мая 2019 г. \\ В окончательной редакции 20 мая 2019 г. \\ Принята к публикации 17 июня 2019 г.
}

\begin{abstract}
Проведены детальные рентгеновские исследования изменения структуры ортобората скандия $\mathrm{ScBO}_{3}$ в процессе последовательных изотермических отжигов исходной шихты в виде аморфного прекурсора. Показано, что при последовательных отжигах аморфного прекурсора первой образуется кристаллическая фаза при температуре отжига, равной $710^{\circ} \mathrm{C}$, положения рефлексов которой не совпадают с положением рефлексов известной кальцитной структуры. Эта фаза существует в очень узком температурном интервале $\left(\approx 30^{\circ} \mathrm{C}\right)$ и при повышении температуры отжига до $750^{\circ} \mathrm{C}$ полностью переходит в известную ромбоэдрическую фазу кальцита. C помощью программного комплекса ENDEAVOUR были определены параметры ячейки, позиции атомов в ячейке и определена пространственная группа симметрии новой фазы. Оказалось, что новая фаза соответствует гексагональной структуре фатерита (S.G. P63/mmc), известной для других ортоборатов редкоземельных элементов (РЗМ).
\end{abstract}

Ключевые слова: наносистемы, бораты РЗМ, фазовые переходы, рентгеновские дифракционные исследования.

DOI: 10.21883/FTT.2019.11.48431.482

\section{1. Введение}

В последние годы большое внимание было уделено редкоземельным ортоборатным люминофорам $R E \mathrm{BO}_{3}$ $(R E=\mathrm{Y}, \mathrm{La}, \mathrm{Pr}, \mathrm{Nd}, \mathrm{Sm}, \mathrm{Eu}, \mathrm{Gd}, \mathrm{Tb}, \mathrm{Dy})$ из-за их интересных люминисцентных свойств, таких как эффективное излучение видимого света под действием рентгеновского излучения, вакуумного ультрафиолета (ВУФ), обычного ультрафиолета (УФ) или инфракрасного (ИК) возбуждения, [1-11]. Эти соединения нашли широкое применение в цветных плазменных панелях и флуоресцентных лампах, не содержащих ртути, [12,13].

Известно, что ортобораты редкоземельных элементов имеют три основные структуры, а именно фатеритную гексагональную структуру с пространственной группой симметрии S.G. P63/mmc (194) $(R E=\mathrm{Y}, \mathrm{Sm}, \mathrm{Eu}, \mathrm{Gd}$, $\mathrm{Tb}, \mathrm{Dy}, \mathrm{Ho}, \mathrm{Er}, \mathrm{Tm}, \mathrm{Yb}, \mathrm{Lu})$, орторомбическую структуру с пространственной группой S.G. Pnam (62) $(R E=\mathrm{La}$, $\mathrm{Ce}, \mathrm{Nd}, \mathrm{Pm})$ и кальцитную ромбоэдрическую структуру с пространственной группой симметрии S.G. $R-3 c$ (167). Кальцитная структура известна только для двух соединений, а именно для $\mathrm{ScBO}_{3}$ и $\mathrm{LuBO}_{3}$, [PDF-2].

Нами ранее были проведены детальные структурные исследования ряда редкоземельных оксидных соединений, синтезированных из аморфного прекурсорного состояния, [14-27]. Было показано, что их структура сильно зависит от размера кристаллитов. Так для соединений $\mathrm{LuBO}_{3}, \mathrm{Eu}_{2}\left(\mathrm{MoO}_{4}\right)_{3}$ и $\mathrm{TbBO}_{3}[14,15,16]$, получаемых из аморфных прекурсоров, осуществляются циклические перестройки структуры с ростом размера кристаллитов.
В этих соединениях при нагреве прекурсора первой кристаллизуется не равновесная для этой температуры, а высокотемпературная фаза. При последующих отжигах, сопровождающихся ростом кристаллитов, эта фаза переходит сначала в равновесную низкотемпературную, известную для микрокристаллического состояния, а при дальнейшем повышении температуры отжига переходит снова в высокотемпературную, но теперь уже равновесную. Основной причиной таких циклических структурных перестроек при синтезе сложных оксидов РЗМ предполагается наличие повышенной энергии атомов на поверхности кристаллитов, процентная доля которых растет с уменьшением размера кристаллитов. В определенных случаях суммарный химический потенциал в таких системах может соответствовать хим. потенциалу высокотемпературной фазы, образование которой и происходит на первых этапах кристаллизации из-за малого размера кристаллитов, образующихся на первых этапах кристаллизации из аморфного прекурсора.

Был также зарегистрирован эффект структурного заражения, когда малые добавки легирующих атомов приводили к образованию структур, свойственных оксидам легирующего элемента, но не образующихся в матричном составе без легирования, [20,21,25]. Так, например, добавки в аморфный прекурсор $\mathrm{YBO}_{3}$, имеющем в кристаллическом состоянии фатеритную гексагональную структуру, атомов $\mathrm{Sc}$, образующих ортоборат $\mathrm{ScBO}_{3}$ с кальцитной структурой, приводило к образованию в $\mathrm{YBO}_{3}$ кальцитной структуры. 
При постановке настоящих исследований мы обратили внимание на то, что, хотя ортоборат лютеция $\mathrm{LuBO}_{3}$ и ортоборат скадия $\mathrm{ScBO}_{3}$ имеют кальцитные фазы, однако фазовые последовательности их сильно отличаются. Так, согласно исследованиям Е.М. Левина (E.M. Levin) с соавторами, [28,29], при твердофазном синтезе ортобората лютеция кальцитной модификации $\mathrm{LuBO}_{3}$ предшествует метастабильная высокотемпературная фатеритная модификация (S.G. P63/mmc), которая образуется при температуре $900^{\circ} \mathrm{C}$ и сохраняется до температуры $1037^{\circ} \mathrm{C}$, а затем переходит в термодинамически равновесную фазу кальцита. Дальнейшее повышение температуры до $1345^{\circ} \mathrm{C}$ приводит к фазовому переходу кальцита в термодинамически равновесную высокотемпературную фазу фатерита. Наши исследования структурных состояний $\mathrm{LuBO}_{3}$ при синтезе из аморфного прекурсорного состояния подтвердили полученные Левиным результаты, [14]. Отличием были температура образования метастабильной фазы фатерита, равная $800^{\circ} \mathrm{C}$, температура перехода из метастабильной фатеритной структуры в равновесную кальцитную структуру, равная $1000^{\circ} \mathrm{C}$, и температура перехода кальцитной модификации в равновесную фатеритную, равная $1250^{\circ} \mathrm{C}$.

Для $\mathrm{ScBO}_{3}$ метастабильная фатеритная фаза, предшествующая фазе кальцита, обнаружена не была не только в стехиометрическом составе, но и при легировании (заражении) аморфного прекурсора $\mathrm{ScBO}_{3}$ атомами $\mathrm{Y}$, образующими ортоборат $\mathrm{YBO}_{3}$ с фатеритной структурой, [21]. На основе этого результата было заключено, что энергия образования кальцитной фазы меньше энергии образования фазы фатерита и отсутствие фазы фатерита на первых этапах кристаллизации $\mathrm{ScBO}_{3}$ из аморфного прекурсора, казалось бы, энергетически обосновано. Однако, как указано выше, при синтезе соединений из аморфных прекурсоров надо учитывать энергетическую добавку поверхностной энергии образующихся нанокристаллитов, которая и приводит к образованию метастабильной высокотемпературной фазы фатерита для $\mathrm{LuBO}_{3}$.

Имея это в виду, мы предположили, что метастабильная фатеритная модификация $\mathrm{ScBO}_{3}$ все же образуется на первых этапах кристаллизации аморфного прекурсора аналогично фазовой последовательности при синтезе $\mathrm{LuBO}_{3}$, но существует в очень узкой температурной области. Поиску такой температурной области образования фатеритной модификации ортобората скандия и посвящены настоящие исследования.

\section{2. Образцы и методы исследования}

Аморфные прекурсоры бората скандия в настоящем исследовании были синтезированы обменным взаимодействием нитратов скандия $\mathrm{Sc}\left(\mathrm{NO}_{3}\right)_{3}$ с борной кислотой $\mathrm{H}_{3} \mathrm{BO}_{3}$ в водной среде. Для синтеза использовали реактивы квалификации ХЧ. Водный раствор нитрата

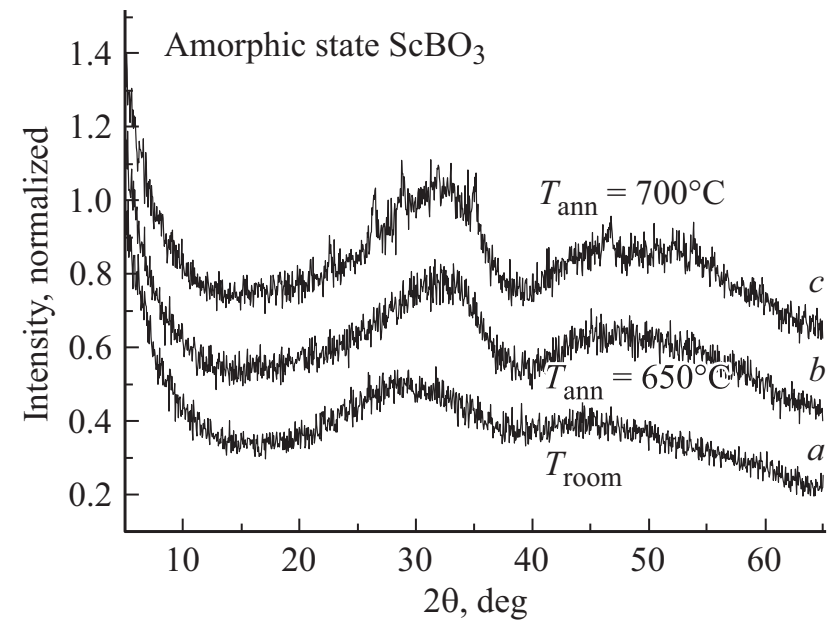

Рис. 1. Трансформации дифракционного рентгеновского спектра аморфного прекурсорного состояния $\mathrm{ScBO}_{3}$ при изменении температуры отжига.

скандия получали либо его растворением в минимальном количестве воды, либо растворением соответствующего количества оксида в 40\%-ой азотной кислоте при кипячении. Далее, водный раствор нитрата смешивали с водным раствором эквимольного количества борной кислоты. При перемешивании добавляли водный раствор аммиака до достижения щелочной реакции среды $(\mathrm{pH}=9.5-10)$. Затем отделяли полученный порошок либо на плотном стеклянном фильтре, либо центрифугированием, промывали несколько раз водой, сушили на воздухе при $50-70^{\circ} \mathrm{C}$ в течение $20 \mathrm{~h}$ и уплотнившуюся компактную массу растирали в агатовой ступке. Структурное состояние образцов регистрировалось на рентгеновском дифрактометре D500 (Siemens) с использованием схемы Брэгга-Брентано. Использовалось монохроматизированное выходным графитовым монохроматором $\mathrm{Cu}-K_{\alpha}$ излучение. Пример температурного изменения дифракционных спектров полученной таким образом аморфной шихты показан на рис. 1.

Твердофазный синтез $\mathrm{ScBO}_{3}$ осуществлялся путем отжига прекурсора при повышенных температурах в лабораторной печи SNOL-6.7/1300 и характеризовался последовательными изотермическими отжигами в течение 2-x h в каждой температурной точке. При этом температурный интервал между последовательными отжигами аморфного состояния составлял $50-100^{\circ} \mathrm{C}$. Монофазное аморфное состояние сохранялось вплоть до температуры отжига, равной $650^{\circ} \mathrm{C}$, см. спектр $(b)$ на рис. 1. Первые изменения галообразного дифракционного спектра были зафиксированы при температуре отжига, равной $700^{\circ} \mathrm{C}$, см. спектр (c) на рис. 1 . Видно, что на спектре появились слабые рефлексы, отвечающие за начальные этапы кристаллизации. Начиная с этого момента, температурный интервал между последовательными отжигами был уменьшен до $10^{\circ} \mathrm{C}$. 


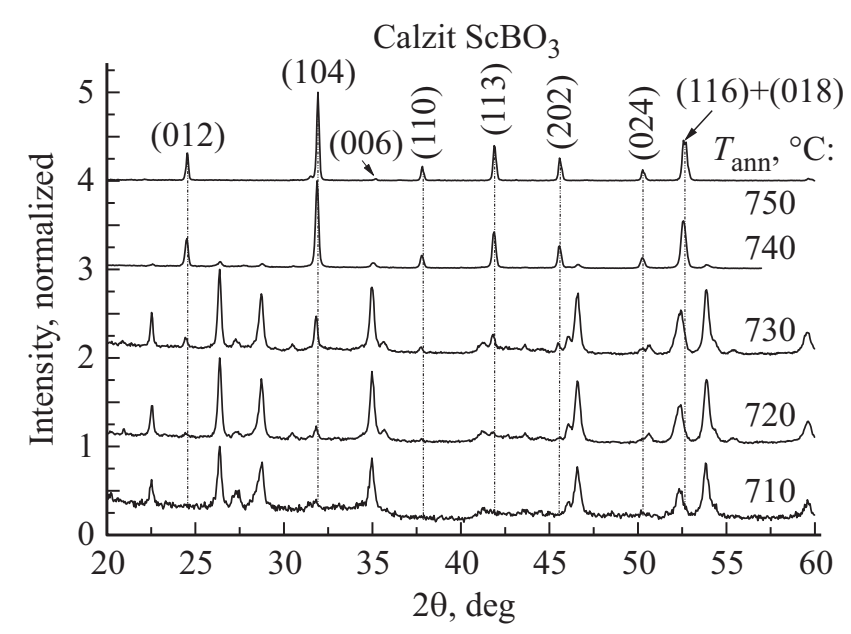

Рис. 2. Трансформация дифракционных спектров $\mathrm{ScBO}_{3}$, начиная с температуры образования кристаллических фаз.

\section{3. Результаты эксперимента и обсуждение}

Трансформация дифракционных спектров $\mathrm{ScBO}_{3}$, начиная с температуры образования кристаллических фаз, показана на рис. 2. Нижний спектр на рис. 2 соответствует температуре отжига прекурсора $T=710^{\circ} \mathrm{C}$ и регистрирует образование первой кристаллической фазы с небольшими остатками аморфной матрицы. Штриховые линии на спектрах рис. 2 соответствуют положениям рефлексов кальцитной модификации $\mathrm{ScBO}_{3}$. Хорошо видно, что отражения, появившиеся при температуре отжига $710^{\circ} \mathrm{C}$ не относятся к кальцитной модификации ортобората скандия. Дальнейшее повышение температуры отжига до $720^{\circ} \mathrm{C}$ привело к тому, что одновременно с ростом интенсивности рефлексов новой кристаллической фазы стали появляться сначала очень слабые, а с повышением температуры отжига до $730^{\circ} \mathrm{C}$ и более интенсивные рефлексы известной кальцитной модификации. При этом интенсивности рефлексов новой фазы значительно увеличились и стали доминирующими на дифракционном спектре. Однако последующий отжиг при $740^{\circ} \mathrm{C}$ привел к катастрофическому падению интенсивностей рефлексов новой кристаллической фазы и значительному росту интенсивностей рефлексов кальцита. После отжига образца при $750^{\circ} \mathrm{C}$ никаких дополнительных рефлексов кроме рефлексов кальцита на дифракционном спектре обнаружено не было, см. рис. 2. Таким образом, новая кристаллическая модификация $\mathrm{ScBO}_{3}$ образуется при отжиге аморфного прекурсорного состояния в температурном интервале между $T=710-740^{\circ} \mathrm{C}$, что составляет всего $\approx 30^{\circ} \mathrm{C}$. Теперь становится понятным, почему такая фаза не была обнаружена ранее. Обычный интервал между последовательными температурами отжига при твердофазном синтезе составляет $\sim 100-200^{\circ} \mathrm{C}$.
Положение атомов в ассимметричной ячейке $\mathrm{ScBO}_{3}$

\begin{tabular}{c|c|c|c|c|c|c}
\hline$n$ & el & chrg & pos & $y$ & $z$ & $x$ \\
\hline 1 & $\mathrm{~B}$ & 3.0 & $6 g$ & 0.50000 & 0.00000 & 0.00000 \\
2 & $\mathrm{Sc}$ & 3.0 & $2 b$ & 0.00000 & 0.00000 & 0.25000 \\
3 & $\mathrm{O}$ & -2.0 & $4 f$ & 0.66667 & 0.33333 & 0.12023 \\
4 & $\mathrm{O}$ & -2.0 & $2 a$ & 0.00000 & 0.00000 & 0.00000
\end{tabular}

Ясно, что температурный интервал в $30^{\circ} \mathrm{C}$ легко может быть пропущен при таких последовательностях изотемпературных отжигов.

Для определения симметрии новой фазы, возникшей на первых этапах кристаллизации мы использовали программу ENDEAVOUR („Structure Solution From Powder Diffraction Data“ (C) Copyright 2000-2015 Crystal Impact, H. Putz \& K. Brandenburg GbR, Authors: Dr. Klaus Brandenburg, Dr. Holger Putz). Использовался дифракционный спектр, полученный при $730^{\circ} \mathrm{C}$, см. рис. 3, и зарегистрированный при минимальном шаге сканирования гониометра, равном $0.02^{\circ}$, и при минимальных параметрах щелевых устройств: расходимость падающего рентгеновского пучка составляла $1^{\circ}$, расходимость регистрирующей щели перед детектором равнялась $0.05^{\circ}$. На рис. 3 индексы, изображенные курсивом относятся к фатеритной модификации, индексы в виде обычного шрифта относятся к нарождающейся фазы кальцита.

Были получены следующие структурные данные:

$$
\begin{gathered}
a=3.8957 \AA, \quad b=3.8957 \AA, \quad c=7.8781 \AA, \\
{\left[\alpha^{\circ}\right]=90.0000, \quad\left[\beta^{\circ}\right]=90.0000, \quad\left[\gamma^{\circ}\right]=120.0000 .}
\end{gathered}
$$

Space-group: $P 63 / m m c . Z=4 . \quad R=0.247$. Положение атомов в ассимметричной ячейке фатеритной структуры показаны в таблице.

Большое значение $R$ фактора обусловлено наличием двухфазного состояния и большим фоном, обусловленным остатками аморфного прекурсора. Тем не менее,

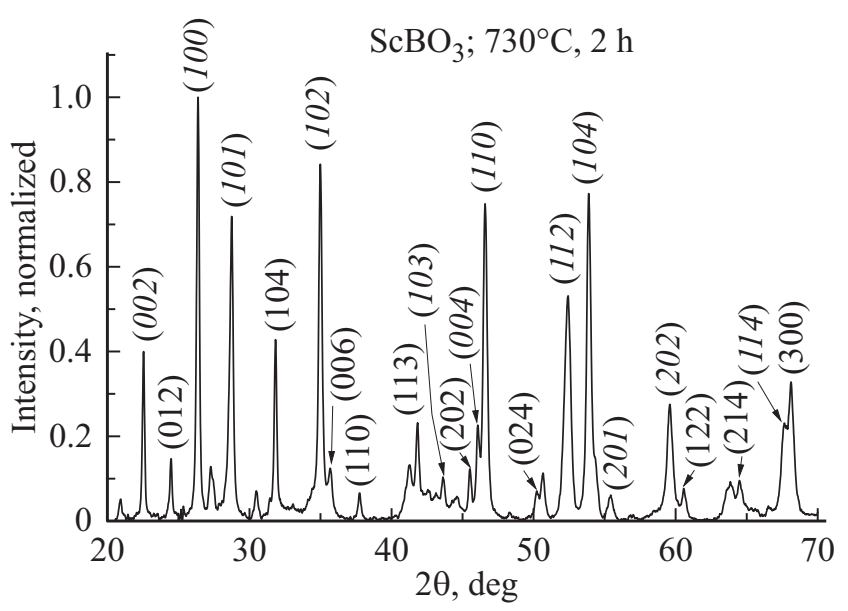

Рис. 3. Детальный дифракционный спектр $\mathrm{ScBO}_{3}$, полученный при отжиге аморфного прекурсора при $730^{\circ} \mathrm{C}$ в течение 2 -х h. 


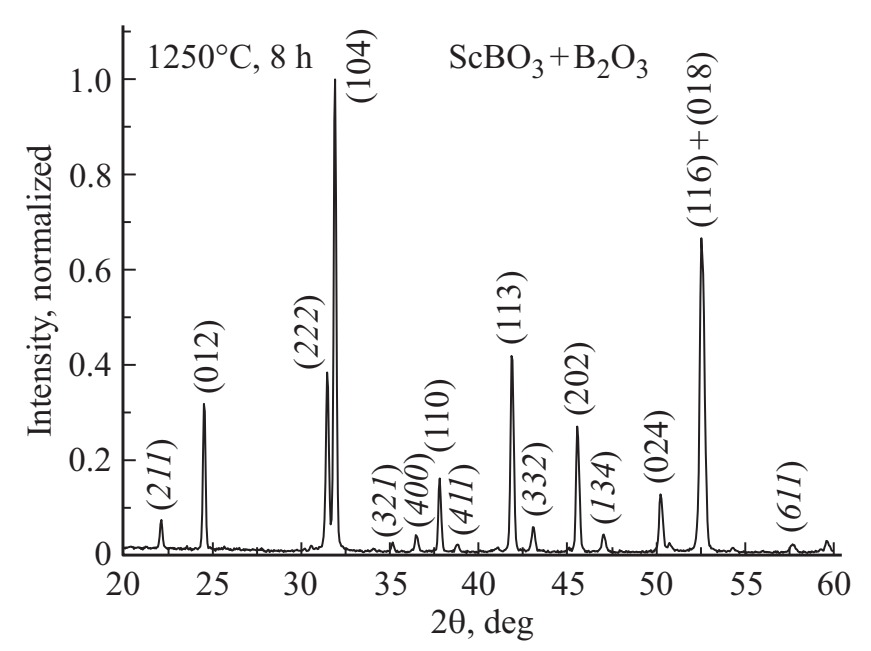

Рис. 4. Дифракционный спектр $\mathrm{ScBO}_{3}$, полученный после длительного отжига образца при температуре $1250^{\circ} \mathrm{C}$.

определенные параметры решетки близки к параметрам фатеритных фаз других редкоземельных ортоборатов, а пространственная группа была устойчива к вариациям параметров итераций определения структуры, что дает нам основание считать полученное решение структуры правильным.

Дальнейшее повышение температуры отжига порошка вплоть до самой высокой температуры используемой печи, равной $1250^{\circ} \mathrm{C}$, не приводило к какимлибо заметным изменениям в числе или положении рефлексов кальцитной модификации, кроме небольшого температурного сдвига центров тяжести, уменьшения полуширины рефлексов и соответственного подрастания интенсивности рефлексов. Однако увеличение времени отжига в несколько раз (вместо 2-х часов до $8 \mathrm{~h}$ ) привело к образованию двухфазного состояния из фазы оксида скандия и остатков кальцитной модификации, см. рис. 4. Начался этап разложения кальцитной структуры.

Возникает очевидный вопрос: „Почему повышение температуры отжига до $1250^{\circ} \mathrm{C}$ не приводит в образованию равновесной фатеритной структуры $\mathrm{ScBO}_{3}$ как в случае ортобората лютеция $\mathrm{LuBO}_{3}$, а приводит к разложению кальцитной модификации до простого оксида скандия?“. Вопрос можно перефразировать следующим образом: „Почему $\mathrm{ScBO}_{3}$ при температуре $1250^{\circ} \mathrm{C}$ разлагается, a $\mathrm{LuBO}_{3}$, не только не разлагается, а остается в кальцитной модификации вплоть до $T=1345^{\circ} \mathrm{C},[28,29]^{*}$. Прежде чем описать возможную модель различия в поведении бората лютеция и бората скандия приведем параметры кристаллических решеток этих структур. Sc: $a=4.748 \AA, c=15.262 \AA, V=297.96 \AA^{3}$. Lu: $a=4.9153 \AA, c=16.212 \AA, V=339.21 \AA^{3}$. Как видно и параметры решетки и объемы ячеек близки. Поскольку структура одна и та же близки и межионные взаимодействия. Что отличает обе структуры, так это масса редкоземельного элемента. Как нам кажется, ответ в различи структурных последовательностей обоих ортоборатов и лежит в соотношении атомных весов $\mathrm{Lu}$ и Sc. Атомная (молярная) масса $\mathrm{Lu}=174.9668 \mathrm{~g} / \mathrm{mol}$, атомная (молярная) масса $\mathrm{Sc}=44.9559 \mathrm{~g} / \mathrm{mol}$. Физическая природа разложения структуры лежит в колебаниях атомов. Когда смещения атомов достигнут определенной величины, они могут покинуть решетку с поверхности кристаллита откуда и начинается любое разложение соединения. Тепловая энергия, приходящаяся на каждый атом, не зависит от типа атома и равна $3 / 2 \kappa T$. Максимальная скорость движения атомов определяется, если приравнять тепловую энергию $3 / 2 \kappa T$ энергии механического движения $W_{\text {kin }}=\left(m \vartheta^{2}\right) / 2$. Отсюда можно получить отношение максимальных скоростей движения $\mathrm{Lu}$ и Sc: $\left(\vartheta_{\mathrm{Sc}} / \vartheta_{\mathrm{Lu}}\right)=\sqrt{(174.9668 / 44.9559)}=1.97$. То есть максимальная скорость Sc почти в два раза больше максимальной скорости Lu. Атомы скандия пролетят в два раза большее расстояние и возможно покинут поверхность кристаллита раньше, нежели это произойдет в борате лютеция. Последнее возможно и определяет температуру разложения бората скандия раньше, нежели произойдет фазовый переход в равновесную фатеритную модификацию.

Можно условно предложить и другой сценарий объяснения разложения $\mathrm{ScBO}_{3}$ при температурах отжига $1200-1250^{\circ} \mathrm{C}$. Если предположить, что, начиная с некоторой высокой температуры отжига, свободная энергия двухфазной системы из кальцита и простого оксида скандия меньше свободной энергии фатеритной структуры, такая система будет стремиться к частичному разложению кальцита. Двухфазной состояние окажется предпочтительным.

\section{4. Заключение}

Показано, что при синтезе ортоборатной структуры $\mathrm{ScBO}_{3}$, получаемой при последовательных изотемпературных отжигах аморфных прекурсоров, образованию известной кальцитной модификации предшествует образование фатеритной структуры, аналогично тому, как это реализуется в ортоборате лютеция. Фатеритная структура образуется в интервале $710-740^{\circ} \mathrm{C}$ и температурный интервал ее существования составляет всего $\sim 30^{\circ} \mathrm{C}$. При температурах отжига выше $740^{\circ} \mathrm{C}$ остается только кальцитная фаза, которая при температуре отжига, равной $1250^{\circ}$, начинает разлагаться на простой оксид $\mathrm{Sc}_{2} \mathrm{O}_{3}$. Таким образом, впервые установлено образование фатеритной структуры $\mathrm{ScBO}_{3}$, предшествующей кальцитной модификации, что аналогично структурным трансформациям $\mathrm{LuBO}_{3}$ как при синтезе из гомогенизированной смеси исходных оксидов, так и при последовательных изотемпературных отжигах аморфного прекурсорного состояния. 


\section{Благодарности}

Автор выражает искреннюю благодарность Г.К. Струковой за помощь в синтезе аморфного прекурсорного состояния $\mathrm{ScBO}_{3}$ и Г.Р. Ганеевой за помощь в определении структуры ортобората скандия.

\section{Финансирование работы}

Работа выполнена в рамках госзадания ИФТТ РАН.

\section{Конфликт интересов}

Автор заявляет, что у него нет конфликта интересов.

\section{Список литературы}

[1] Z. Wei, L. Sun, C. Liao, J. Yin, X. Jiang, C. Yan, S. Lü. J. Phys. Chem. B 106, 10610 (2002).

[2] H. Giesber, J. Ballato, W. Pennington, J. Kolis. J. Inf. Sci. 149, $61(2003)$

[3] H. Giesber, J. Ballato, G. Chumanov, J. Kolis, M. Dejneka. J. Appl. Phys. 93, 8987 (2003).

[4] T. Kim, S. Kang. Mater. Res. Bull. 40, 1945 (2005).

[5] J. Lin, Y. Huang, J. Zhang, X. Ding, S. Qi, C. Tang. Mater. Lett. 61, 1596 (2007).

[6] L. Chen, Y. Jiang, S. Chen, G. Zhang, C. Wang, G. Li. J. Lumin. 128, 12, 2048 (2008).

[7] J. Dexpert-Ghys, R. Mauricot, B. Caillier, P. Guillot, T. Beaudette, G. Jia, P. Tanner, B. Cheng. J. Phys. Chem. C 114, 14, 6681 (2010).

[8] J. Li, Y. Wang, B. Liu. J. Lumin. 130, 981 (2010).

[9] A. Szczeszak, S. Lis, V. Nagirnyi. J. Rare Earths 29, 12, 1142 (2011).

[10] Z. Yang, D. Yan, K. Zhu, Z. Song, X. Yu, D. Zhou, Z. Yin. J. Mater. Lett. 65, 8, 1245 (2011).

[11] A. Szczeszak, T. Grzyb, S. Lis, R. Wiglusz. J. Dalton Trans. 41, 19, 5824 (2012).

[12] S. Choi, B.-Y. Park, H.-K. Jung. J. Lumin. 131, 1460 (2011).

[13] C.R. Ronda, T. Jüstel, H. Nikol. J. Alloys Compd. 275-277, 669 (1998).

[14] I.M. Shmytko, G.K. Strukova, E.A. Kudrenko. Crystallogr. Rep. 51, Suppl. 1, S163 (2006).

[15] А.П. Киселев, С.3. Шмурак, Б.С. Редькин, В.В. Синицын, И.М. Шмытько, Е.А. Кудренко, Е.Г. Понятовский. ФТТ 48, 1458 (2006).

[16] I.M. Shmytko. Phys. Solid State 61, 207 (2019).

[17] Е.А. Кудренко, И.М. Шмытько, Г.К. Струкова. ФТТ 50, 924 (2008).

[18] И.М. Шмытько, Е.А. Кудренко, Г.К. Струкова, Н.В. Классен. ФТТ 50, 1108 (2008).

[19] И.М. Шмытько, Г.К. Струкова. ФТТ 51, 1796 (2009).

[20] I. Shmytko, G. Strukova, E. Kudrenko. Acta Cryst. A 66, S230 (2010)

[21] I.M. Shmytko, I.N. Kiryakin, G.K. Strukova. Phys. Solid State 53, 377 (2011)

[22] I. Shmytko. Acta Cryst. A 67, C533 (2011).

[23] V.V. Kedrov, I.M. Shmyt'ko, S.Z. Shmurak, E.A. Kudrenko, N.V. Klassen. J. Mater. Res. 27, 2117 (2012).

[24] И.М. Шмытько, И.Н. Кирякин, Г.К. Струкова. ФТТ 55, 1364 (2013).
[25] И.И. Шмытько, И.Н. Кирякин, Г.К. Струкова. ФТТ 55, 1369 (2013).

[26] E.A. Kudenko, I.M. Shmytko, G.K. Strukova. Acta Cryst., A 64, C427 (2008).

[27] I.M. Shmytko, E.A. Kudrenro, G.K. Strukova, V.V. Kedrov, N.V. Klassen. Z. Kristallogr. Suppl. 27, 211 (2008).

[28] E.M. Levin, R.S. Roth, J.B. Martin. Am. Mineral. 46, 9-10, 1030 (1961).

[29] E.M. Levin. In Phase Diagrams / Ed. A.M. Alper Academic, N.Y. 3, 180 (1970).

Редактор Т.Н. Василевская 